\title{
Os benefícios da equoterapia no equilíbrio de um paciente com sequelas de acidente vascular encefálico
}

\author{
The benefits of equotherapy in the equilibrium of a patient \\ with sequels of stroke
}

\section{Los beneficios de la equoterapia en equilibrar a un paciente con secuencias de accidente vascular cerebrale}

\author{
Aimê Cunha Arruda ${ }^{1}$, Magali Kellermann da Silva², Lia da Porciuncula \\ Dias da Costa ${ }^{3}$
}

1.Mestranda em Atenção Integral à Saúde (Unicruz/Unijuí), bacharel em Fisioterapia (Unicruz). Cruz Alta-RS, Brasil.

2. Bacharel em Fisioterapia (Unicruz). Cruz Alta-RS, Brasil.

3. Mestre em Educação nas Ciências, professora adjunta da Universidade de Cruz Alta e orientadora da pesquisa. Cruz Alta-RS, Brasil.

\begin{abstract}
Resumo
Objetivo. Avaliar os benefícios da equoterapia no equilíbrio de um paciente com sequelas de acidente vascular encefálico. Método. A pesquisa tem a característica de um estudo de caso longitudinal prospectivo, qualitativo e quantitativo, exploratório descritivo. Foram utilizadas como método 3 avaliações: pré-avaliação, tendo como base os dados da Escala de Equilíbrio de Berg, no CEEASA, ao final de 30 atendimentos no ano de 2017. Em seguida, o sujeito ficou um período de 3 meses sem atendimentos. Em março de 2018, foi realizada a avaliação inicial no Centro de Equoterapia Unicruz - CEU, e a avaliação final, após 10 atendimentos de equoterapia, uma vez por semana, com duração de 30 minutos. Os dados foram analisados através de pacote estatístico adequado. Resultados. Verificou-se que 0 equilíbrio da praticante após a terapia alcançou ganho de 7 pontos na escala total (48-55). Conclusão. A equoterapia mostrou-se um tratamento complementar e contínuo, pois sem ela o praticante regride em suas aptidões.
\end{abstract}

Unitermos. Reabilitação; fisioterapia; acidente vascular cerebral

\begin{abstract}
Objective. To evaluate the benefits of hippotherapy on the balance of a patient with stroke sequelae. Method. The research was characterized by a prospective, qualitative and quantitative, exploratory descriptive longitudinal case study. Three evaluations were used as the method: pre-assessment, based on data from the Berg Balance Scale at CEEASA, at the end of 30 consultations in 2017. After this, the subject had a period of 3 months without consultations. In March 2018, the initial evaluation was performed at the Unicruz Equine Therapy Center - CEU, and the final evaluation, after 10 equine therapy visits, once a week, lasting 30 minutes. Data were analyzed using appropriate statistical package. Results. It was found that the practitioner's balance after therapy achieved a gain of 7 points on the full scale (48-55). Conclusion. Equine therapy was a complementary and continuous treatment, because without it the practitioner regresses in their skills.
\end{abstract}

Keywords. Rehabilitation; physiotherapy; stroke

\section{Resumen}

Objetivo. evaluar los beneficios de la hipoterapia en el equilibrio de un paciente con secuelas de accidente cerebrovascular. Método. La investigación se caracterizó por un estudio de caso longitudinal descriptivo exploratorio prospectivo, cualitativo y cuantitativo. Se utilizaron tres evaluaciones como método: preevaluación, basada en datos de la Escala de Balance de Berg en CEEASA, al final de 30 consultas en 2017. Después de esto, el sujeto 
tuvo un período de 3 meses sin consultas. En marzo de 2018, la evaluación inicial se realizó en el Centro de Terapia Equina Unicruz - CEU, y la evaluación final, después de 10 visitas de terapia equina, una vez a la semana, con una duración de 30 minutos. Los datos se analizaron utilizando el paquete estadístico apropiado. Resultados. se encontró que el equilibrio del profesional después de la terapia logró una ganancia de 7 puntos en la escala completa (48-55). Conclusión. la terapia equina era un tratamiento complementario y continuo, porque sin ella el profesional retrocede en sus habilidades.

Palabras clave. Rehabilitación; fisioterapia; accidente cerebrovascular

Trabalho realizado na Universidade de Cruz Alta (UNICRUZ), Cruz Alta-RS, Brasil.

\section{INTRODUÇÃO}

Segundo a Organização Mundial de Saúde (OMS), desde a década de 1970, as doenças cerebrovasculares são a primeira causa básica de morte no mundo. Devido ao aumento da expectativa de vida e mudanças no estilo de vida da população, o Acidente Vascular Encefálico (AVE) está se tornando cada vez mais comum ${ }^{1}$.

A fraqueza, os movimentos sinérgicos anormais e a espasticidade resultam em um padrão de marcha alterado, o que contribui para uma dificuldade de equilíbrio, risco de quedas e aumento do consumo de energia durante a caminhada. Portanto, a recuperação da marcha requer diferentes técnicas que vão exigir a capacidade do fisioterapeuta de auxiliar os pacientes a suportar seu próprio peso corporal e controlar seu equilíbrio².

Com base nesses aspectos, a marcha do cavalo dará ao praticante movimentos rítmicos e repetitivos semelhantes à caminhada do ser humano, contribuindo para a melhora da postura, equilíbrio e força ${ }^{3}$. Assim, o 
estímulo proprioceptivo irá contribuir para o amadurecimento sensório-motor do paciente, pois ele fará uma reorganização postural a cada movimento do cavalo, buscando equilíbrio ${ }^{4}$.

A partir disso, o objetivo geral da pesquisa foi avaliar os benefícios da equoterapia no equilíbrio de um paciente com sequelas de AVE.

\section{MÉTODO}

\section{Amostra}

De acordo com a Resolução n० 466/12 do Conselho Nacional de Saúde, que regulamenta as pesquisas envolvendo seres humanos, a participação na presente pesquisa foi voluntária, o paciente apresentou condições cognitivas e físicas de assinar o Termo de Consentimento Livre e Esclarecido. Esta pesquisa foi submetida ao Comitê de Ética e Pesquisa (CEP) da Universidade Cruz Alta Centro das Ciências da Saúde e Agrárias - Cruz Alta, RS, Brasil, e aprovado sob o número do parecer 2.625.452.

Esta pesquisa apresentou característica de um estudo de caso longitudinal prospectivo, sob perspectiva qualitativa e quantitativa, exploratória e descritiva. O sujeito do estudo já participava dos atendimentos como praticante do Centro de Equoterapia EASA/UNICRUZ, dentro do projeto de extensão CEEASA-UNICRUZ, GAP00659/20170403172153. A seleção ocorreu de acordo com a ordem de inscrição e disponibilidade de horário, diagnóstico e indicação médica, 
bem como avaliações dos profissionais das áreas envolvidas respondendo a uma ficha de informações própria do Centro. Em virtude do encerramento do convênio com a EASA, em dezembro de 2017, a continuidade da pesquisa ocorreu em março de 2018, no Centro de Equoterapia UNICRUZ - CEU, que possui cadastro dentro do gabinete de projetos de extensão

aprovados

pela

instituição:

GAP00738/20171011152409.

\section{Procedimento}

A partir da avaliação multiprofissional, foi elaborada a atuação durante a equoterapia, pensando nos objetivos propostos da pesquisa. No início de cada atendimento, eram realizados alongamentos de membros superiores e inferiores ativos assistidos. Após, a praticante era conduzida até a rampa para montar no cavalo, com o auxílio de um terapeuta na rampa e outro como guia.

Durante a montaria, foram realizadas mobilizações ativo-assistidas, alongamento global, dissociação de cinturas escapular e pélvica, aliado ao movimento tridimensional e rotacional do cavalo, com o auxílio de um guia que conduzia o cavalo e um terapeuta como apoio lateral, para a orientação das atividades e também para segurança da praticante.

Foram realizadas 3 avaliações: pré-avaliação, na qual utilizamos como base os dados da Escala de Equilíbrio de Berg (EEB), no CEEASA, ao final de 30 atendimentos no ano de 2017. Em seguida, o sujeito ficou um período de 3 
meses sem atendimentos de equoterapia e fisioterapia. Em março de 2018, foi realizada a avaliação inicial, quando buscamos verificar se ocorreram alterações no quadro clínico após o período sem atividades equoterapêuticas e identificar efeitos residuais dos ganhos obtidos anteriormente. E a avaliação final, que foi realizada após 10 atendimentos de equoterapia, na frequência de uma vez por semana, com duração de 30 minutos montado. Utilizouse a andadura ao passo. A escala de avaliação utilizado para verificar os benefícios da equoterapia foi a EEB.

A EEB é uma escala ordinária baseada no desempenho, que avalia a capacidade do indivíduo para manter o equilíbrio durante o desempenho de 14 tarefas comuns no cotidiano. O desempenho de cada tarefa é avaliado em uma escala ordinal de 5 pontos, variando de 0 a 4, de acordo com a qualidade do movimento ou o tempo gasto para realizá-lo. O ponto 0 (zero) representa a necessidade de assistência máxima, e 4 pontos indicam que o indivíduo é funcionalmente independente para executar a tarefa. Uma pontuação total (máximo de 56 pontos) é calculada pela soma de cada item. Índices mais altos revelam uma melhor capacidade de manter um bom equilíbrio ${ }^{5}$.

O sujeito da pesquisa foi um praticante do sexo feminino, RPS, 38 anos, com sequelas de AVE ocorrido há 4 anos. Nesse período apresentou quadro temporário de estresse emocional, hipertensão arterial, convulsão, cegueira e afasia, permanecendo 28 dias hospitalizada. 
Possui um quadro clínico de hemiparesia do hemicorpo esquerdo, levando a uma marcha do tipo ceifante.

O sujeito da pesquisa continuou no projeto de extensão do CEU depois da finalização do estudo, não interferindo na sua permanência.

\section{Análise Estatística}

Os dados foram analisados através de pacote estatístico adequado, sendo apresentados por meio de frequência e percentual, os dados quantitativos através de medidas centrais e dispersão (média e desvio padrão) e apresentados em formato de tabela.

\section{RESULTADOS}

Através da avaliação com a EEB, foi possível verificar que o equilíbrio da praticante após o treinamento de 10 sessões de equoterapia alcançou ganho de 7 pontos na escala total (48-55). Na pré-avaliação, obteve um resultado de 53 pontos. Na avaliação inicial, apresentou uma perda de equilíbrio de 5 pontos (48), principalmente nos seguintes itens: apanhar objeto do chão, girando 360 graus, colocar os pés alternadamente sobre um banco, em pé com um pé em frente ao outro, em pé apoiado em um dos pés. Já na avaliação final, atingiu um ganho na capacidade de equilíbrio com 55 pontos, proporcionando a recuperação da perda e o benefício da terapia (Tabela 1 ). 
Tabela 1. EEB.

\begin{tabular}{|c|c|c|c|c|c|}
\hline Descrição dos itens & $\begin{array}{c}\text { Pré- } \\
\text { Avaliação }\end{array}$ & $\begin{array}{c}\text { Avaliação } \\
\text { Inicial }\end{array}$ & $\begin{array}{c}\text { Avaliação } \\
\text { Final }\end{array}$ & Destreino & $\begin{array}{l}\text { Benefício } \\
\text { da terapia }\end{array}$ \\
\hline 1. Sentado para em pé & 4 & 4 & 4 & & \\
\hline 2. Em pé sem apoio & 4 & 4 & 4 & & \\
\hline 3. Sentado sem apoio & 4 & 4 & 4 & & \\
\hline 4. Em pé para sentado & 4 & 4 & 4 & & \\
\hline 5. Transferências & 3 & 3 & 4 & Não & Sim \\
\hline $\begin{array}{l}\text { 6. Em pé com os olhos } \\
\text { fechados }\end{array}$ & 4 & 4 & 4 & & \\
\hline 7. Em pé com os pés juntos & 4 & 4 & 4 & & \\
\hline $\begin{array}{l}\text { 8. Reclinar à frente com os } \\
\text { braços estendidos }\end{array}$ & 4 & 4 & 4 & & \\
\hline 9. Apanhar objeto do chão & 4 & 3 & 4 & Sim & Sim \\
\hline $\begin{array}{l}\text { 10. Virando-se para olhar para } \\
\text { trás }\end{array}$ & 3 & 4 & 4 & Não & \\
\hline 11. Girando 360 graus & 4 & 2 & 4 & Sim & Sim \\
\hline $\begin{array}{l}\text { 12. Colocar os pés } \\
\text { alternadamente sobre um } \\
\text { banco }\end{array}$ & 4 & 3 & 4 & Sim & Sim \\
\hline $\begin{array}{l}\text { 13. Em pé com um pé em } \\
\text { frente ao outro }\end{array}$ & 3 & 2 & 3 & Sim & Sim \\
\hline $\begin{array}{l}\text { 14. Em pé apoiado em um dos } \\
\text { pés }\end{array}$ & 4 & 3 & 4 & Sim & Sim \\
\hline Total & 53 & 48 & 55 & & \\
\hline
\end{tabular}

\section{DISCUSSÃO}

A hemiparesia é definida como um sinal esperado em pacientes pós-AVE, manifestando-se pela paralisia motora parcial do hemicorpo contralateral ao hemisfério lesado, devido à lesão do neurônio motor superior. Neste contexto, os pacientes com lesão de Hemisfério Cerebral Direito (HCD) apresentam menor equilíbrio postural sentado e em pé, com prejuízo da imagem corporal e heminegligência ${ }^{4}$, quadro clínico este observado no sujeito do estudo.

Comprova-se a eficácia da terapia na reabilitação de pacientes com sequelas de AVE já que estes apresentam dificuldade de equilíbrio e controle postural para em pé, 
sendo prejudicados pela postura assimétrica, desequilíbrio corporal anormal e déficit de transferência de peso ${ }^{6}$.

Por meio dos movimentos executados, é possível o fisioterapeuta trabalhar, durante a equoterapia, o equilíbrio de tronco, a melhora da postura e da lateralidade, o esquema corporal, as atividades viso-espaciais, a psicomotricidade fina e a grossa, a disciplina e outras tantas aquisições necessárias também à aprendizagem² ${ }^{2}$.

Os atendimentos foram realizados com total segurança. Antes de iniciar e durante a montaria é de extrema importância a adaptação do paciente a essa nova situação, sendo que os profissionais envolvidos no atendimento devem ter cautela no momento do ato de montar, relacionando essa atividade a um ato de prazer e descontração ${ }^{7}$.

A terapia fornece ao paciente montado estímulos proprioceptivos que auxiliam para amadurecimento sensório-motor, proporcionando uma organização postural a cada deslocamento do animal, com isso o paciente busca o equilíbrio e novas estratégias motoras devido às diferentes posturas impostas ${ }^{8}$.

Durante uma sessão de 30 minutos, os pacientes experimentam cerca de 2700 a 3300 repetições de desafio postural obrigatórias, pois os movimentos do cavalo geram entradas vestibulares contínuas que causam ao praticante uma adaptação constante a esses movimentos, obtendo reações para corrigir o reequilíbrio através do estímulo e 
proporcionando a melhora do controle postural e dos músculos do tronco?.

A equoterapia utiliza impulsos motores gerados nas costas de um cavalo enquanto este está andando. Os impulsos locomotores são os efeitos do seu movimento durante a caminhada e são transformados no corpo do cavaleiro a uma frequência de 90 a 110 impulsos por minuto $(1,5$ a $1,8 \mathrm{~Hz})$ em 3 planos de movimento. Estes impulsos surgem da decolagem dos membros posteriores e do impacto dos membros frontais do cavalo. O movimento forçado e dinâmico do cavalo interfere mais no equilíbrio dinâmico do que no equilíbrio estático do paciente ${ }^{10}$.

Os pacientes adquirem a recuperação do equilíbrio postural através da adaptação a essa informação. Por todas essas vantagens e semelhança com a marcha humana, a terapia tem efeitos consideráveis no desempenho e no equilíbrio, maiores do que outros treinamentos já estudados, sendo um método de tratamento útil para pacientes com AVE ${ }^{11}$.

Encontramos resultados semelhantes em estudo que relatou uma melhora gradual do equilíbrio postural dos praticantes, pela redução significativa no deslocamento médio-lateral e anteroposterior após 12 sessões de equoterapia, porém após o período de interrupção de 45 dias, os deslocamentos aumentaram, gerando maior oscilação no sentido médio-lateral e anteroposterior, indicando que a interrupção do tratamento pode causar uma deterioração no equilíbrio postural ${ }^{8}$. 
O mesmo estudo verificou que após o retorno à terapia houve uma redução adicional no deslocamento, demonstrando que a retomada do tratamento melhorou novamente o equilíbrio postural. Isso pode indicar que mais sessões têm melhores efeitos no equilíbrio postural, evidenciando relevância da terapia continuada ${ }^{8}$.

O praticante responde seguidamente a um ambiente em mudança, e isso promove comportamentos adaptativos, reações posturais automáticas, estratégias de movimento, respostas antecipatórias e feedback de controle postural. Tais reações de equilíbrio são induzidas na tentativa de mantê-lo através dos distúrbios causados pelo movimento rítmico e repetitivo do cavalo ${ }^{12}$.

Transferência de peso, capacidade de ficar em pé sem apoio, andar sem o auxílio, parar e girar com sucesso, avaliados com EEB, são aquisições compatíveis com outro estudo, a equoterapia facilita as reações de equilíbrio, o que contribui para a base do movimento típico e habilidades funcionais necessárias ao praticante ${ }^{2}$.

Nossos resultados vão ao encontro dos seguintes estudos, os quais discutiram a influência da equoterapia na marcha e equilíbrio de indivíduos hemiparéticos pós-AVE, avaliados através da EEB, melhorando a velocidade da marcha e a relação de assimetria do comprimento do passo, sendo um tratamento útil para pacientes com $\operatorname{AVE}^{10,12}$.

Observou-se que nos primeiros 2 atendimentos utilizamos a sela inglesa como prática de enduro para a 
equoterapia, proporcionando mais estabilidade pélvica, porém a praticante relatou um quadro de lombalgia, e decidimos ao invés da sela utilizar a manta para os 8 atendimentos restantes, proporcionando deslocamento pélvico adequado, pelo movimento de báscula da cintura pélvica, levando a uma mobilização articular.

Em função desse quadro álgico, resolvemos realizar ao final do atendimento manobras de liberação miofascial na coluna cervical e lombar, como técnicas de pompage, o que promoveu alívio imediato da dor, sendo possível suspender nas demais sessões.

Para evoluir de uma marcha compensatória para uma típica, estudos com métodos de tratamento capazes de melhorar a marcha e a capacidade de equilíbrio de pacientes com AVE devem ser conduzidos. Este estudo encontrou melhora na velocidade da marcha, bem como no escore do Berg, demonstrando que a equoterapia não apenas aumenta a velocidade e o comprimento do passo, mas também melhora o equilíbrio, reduzindo o risco de queda ${ }^{3,13}$.

\section{CONCLUSÃO}

Foi possível concluir, com esta pesquisa, que a equoterapia promoveu equilíbrio, alinhamento postural e adequação do tônus muscular espástico ao praticante. As atividades executadas na avaliação com EEB como, apanhar objeto do chão, capacidade de ficar em pé sem 
apoio, girar 360 graus, colocar os pés alternadamente sobre um banco, um pé em frente ao outro e apoio em um dos pés, evidenciaram o destreino pela interrupção da terapia na avaliação inicial, enquanto que na avaliação final a houve a recuperação das aquisições aliada ao benefício da terapia.

\section{AGRADECIMENTOS}

Ao Centro de Equoterapia Unicruz - CEU, Universidade de Cruz Alta, Projeto Pibex CEU.

\section{REFERÊNCIAS}

1.Silva Filho EM, Albuquerque JA. Influência da terapia de restrição e indução do movimento no desempenho funcional de pacientes com acidente vascular encefálico: um ensaio clínico randomizado. Fisioter Pesqui 2017;24:180-90. http://dx.doi.org/10.1590/18092950/16874424022017

2.Beinotti F, Correia N, Christofoletti G, Borges G. Use of hippotherapy in gait training for hemiparetic post-stroke. Arq Neuropsiquiatr 2010;68:908-13.

http://dx.doi.org/10.1590/S0004-282X2010000600015

3.Lee CW, Kim SG, Yong MS. Effects of hippotherapy on recovery of gait and balance ability in patients with stroke. J Phys Ther Sci 2014;26:309-11. http://dx.doi.org/10.1589/jpts.26.309

4.Pedebos BM, Porto LB, Copetti F, Balk RS. Avaliação do controle postural e sua relação com o hemisfério acometido em pacientes com acidente vascular cerebral praticando equoterapia. Fisioter Bras 2014;15:22-8.

http://www.portalatlanticaeditora.com.br/index.php/fisioterapiabrasil/ article/view/308/532

5.Ghotbi N, Nakhostin Ansari N, Naghdi S, Hasson S. Measurement of lower-limb muscle spasticity: intrarater reliability of modified modified ashworth scale. J Rehabil Res Dev 2011;48:83-8. http://dx.doi.org/10.1682/jrrd.2010.02.0020

6.Bunketorp-Käll L, Lundgren-Nilsson $\AA$, Samuelsson $H$, Pekny $\mathrm{T}$, Blomvé $\mathrm{K}$, Pekna $\mathrm{M}$, et al. Long-term improvements after 
multimodal rehabilitation in late phase after stroke a randomized controlled trial. Stroke 2017;48:1916-24.

http://dx.doi.org/10.1161/STROKEAHA.116.016433

7.Costa LPD. A equoterapia como forma de inclusão social para o portador de necessidades especiais. In: Adams A, Brutti TA, Peranzoni VC (Orgs). Inclusão social e preconceitos na contemporaneidade - Volume II. Curitiba: Editora CRV, 2016.

8. Moraes AG, Copetti F, Ângelo VR, Chiavoloni L, de David AC. Hippotherapy on postural balance in the sitting position of children with cerebral palsy - Longitudinal study. Physiother Theory Pract 2018,11:1-8. https://doi.org/10.1080/09593985.2018.1484534 9.Chung EJ, Kim JH, Lee BH. The Effects of Core Stabilization Exercise on Dynamic Balance and Gait Function in Stroke Patients. J Phys Ther Sci 2013;25:803-6. https://doi.org/10.1589/jpts.25.803

10. Han JY, Kim JM, Kim SK, Chung JS, Lee HC, Lim JK, et al. Therapeutic effects of mechanical horseback riding on gait and balance ability in stroke patients. Ann Rehabil Med 2012;36:762-9. https://doi.org/10.5535/arm.2012.36.6.762

11. Maćków A, Małachowska-Sobieska M, Demczuk-Włodarczyk E, Sidorowska M, Szklarska A, Lipowicz A. Influence of neurophysiological hippotherapy on the transference of the centre of gravity among children with cerebral palsy. Ortop Traumatol Rehabil 2014;16:581-93. https://doi.org/10.5604/15093492.1135048

12.Bondia JP, Ferreira RCC, Muniz GMM. Equoterapia no controle postural em pacientes com hemiparesia espástica após AVE. Fisioterapia Brasil 2014;15:1-8.

http://porteiras.s.unipampa.edu.br/gpfin/files/2014/06/artigo-4-

Fisioterapia-Brasil- v15n1-Rodrigo-de-Souza-Balk.pdf

13. Hess D, Nishiwaki J, Liberatori MF, Eichinger FLF, Soares AV. Modalidades de tratamento da espasticidade. Cad Educ Saúde Fisioter 2017;4:46-56. https://doi.org/10.18310/23588306.v4n7.p46 